Original Paper http://ajol.info/index.php/ijbcs http://indexmedicus.afro.who.int

\title{
Connaissance des OGMs et canaux d'information des acteurs au Burkina Faso
}

\author{
Jean SIMPORE ${ }^{1}$, Ouango Maurice SAVADOGO ${ }^{2,3^{*}}$, Moussa SAVADOGO $^{4}$ et \\ Dapola Evariste Constant DA ${ }^{1}$ \\ ${ }^{1}$ Laboratoire Dynamique des espaces et sociétés, Université Ouagal Pr Joseph Ki Zerbo, 03BP: 7021 \\ Ouagadougou, Burkina Faso. \\ ${ }^{2}$ Institut de l'Environnement et de Recherches Agricoles, 04 BP: 8645 Ouagadougou 04, Burkina Faso. \\ ${ }^{3}$ Université Ouaga 1, Unité de Formation et de Recherche en Sciences de la Vie et de la Terre, Laboratoire de \\ Sciences du Sol et de l'Environnement, O3BP: 7021 Ouagadougou, Burkina Faso. \\ ${ }^{4}$ African Biosafety Network of Expertise, Burkina Faso. \\ *Auteur correspondant ; E-mail : savadogoouango@yahoo.fr; Tel :00 22660646562.
}

\section{RESUME}

Pour subvenir aux besoins d'une population en croissance, les scientifiques proposent toujours de nouvelles technologies de production. Ces technologies ne sont pas toujours acceptées pour des raisons défendues par les groupes d'acteurs en présence. A la recherche de solutions pour améliorer la productivité agricole, les autorités du Burkina Faso se sont tournées vers l'utilisation des organismes génétiquement modifiés (OGM). L'étude vise à appréhender la connaissance des OGM et les principaux canaux d'information au Burkina Faso. Pour la réalisation de cette étude, une enquête a été conduite auprès de 272 personnes réparties dans 16 communes de trois provinces du Burkina Faso. L'étude a montré que sans discrimination du niveau d'instruction ou du genre, les acteurs ont une certaine connaissance des OGM. Les principaux canaux d'information sont le réseau d'encadrement des sociétés cotonnières (42\%) et Internet (34\%). Les réseaux d'encadrement sont effectifs dans la diffusion de l'information technologique. Dans la présente étude, il ressort que les technologies de communication sont efficaces pour une diffusion plus large.

() 2016 International Formulae Group. All rights reserved.

Mots clés : Burkina Faso ; OGM ; canaux d'information; niveau d'instruction.

\section{Stakeholders GMOs knowledge and information channels in Burkina Faso}

\begin{abstract}
To meet the needs of a growing population, scientists propose new production technologies. These technologies are not always accepted for reasons defended by stakeholders. Seeking solutions to improve agricultural productivity, the Burkina Faso authorities decided to take advantage of genetically modified organisms (GMO). The study aimed to understand the knowledge of GMOs and the main channels of information in Burkina Faso. To carry out this study, a survey was conducted with 272 individuals in 16 communes in three provinces of Burkina Faso. The study showed that without education and gender based discrimination, the stakeholders have basic knowledge of GMOs. The main information channels are the cotton companies' extension network (42\%) and Internet (34\%). Extension networks are effective in disseminating
\end{abstract}


technological information. In this study, it appears that communication technologies are effective for wider dissemination.

(C) 2016 International Formulae Group. All rights reserved.

Keywords: Burkina Faso; GMOs; information channels; Level of instruction.

\section{INTRODUCTION}

L'agriculture est un secteur important et elle doit relever le défi face à la démographie galopante et au changement climatique (Kahn, 2005; Umezawa et al., 2006; Griffon, 2010). Au Burkina Faso, c'est un domaine qui occupe plus de $80 \%$ de la population. Ce secteur majeur d'activité connait des difficultés biotiques (notamment les attaques des ravageurs et les mauvaises herbes) et abiotiques qui incluent les sécheresses, la pauvreté des sols (Gnankiné et al., 2007; Koulibaly et al., 2013; Houndete et al., 2015; Koulibaly et al., 2016). Ces difficultés plombent le développement du secteur agricole avec la réduction de la productivité et la rentabilité des cultures vivrières et de rente (Bourgou et al., 2014).

Ainsi, pour réduire les coûts de production, les autorités ont toujours apporté des solutions en explorant certaines technologies (Ouattara, 2011; Degla, 2012). Parmi ces technologies figurent les Organismes Génétiquement Modifiés (OGM) qui reste peu connus (Rule et Langa, 2005) et dont l'usage soulève des controverses. Avec tout ce que cette technologie peut comporter comme inconvénients, le Burkina Faso, fut le premier pays d' "Afrique Noire » a autorisé la commercialisation des OGM, à savoir le coton Bt (Rule et Langa, 2005; ChaklattiRousseliere, 2006). Quelle connaissance les populations ont des OGM? Comment les populations reçoivent-ils les informations sur les OGM ? Cet article a investigué sur les connaissances des OGM et les principaux canaux d'information. Dans cet article, nous donnons les différents canaux de réception d'information et les connaissances des populations sur ces OGM.

\section{MATERIEL ET METHODES \\ Approche méthodologique et collecte des données}

Deux principaux groupes d'acteurs que sont les producteurs et les utilisateurs ont été considérés par l'étude : le groupe concerne les producteurs (186), celui des autres utilisateurs composés des enseignants des universités et les chercheurs (19) et des utilisateursconsommateurs (67). L'enquête s'est déroulée dans trois provinces, 16 communes et 30 villages. Les enseignants et les chercheurs sont supposés être en avance dans le domaine des évolutions scientifiques, techniques et technologiques.

L'étude a couvert quatre zones qui sont représentatives des grands ensembles sur la base des potentialités biophysiques, de production et de consommation. Les producteurs ont été identifiés dans trois provinces qui sont le Bam, le Gourma et le Houet. Les autres utilisateurs ont été pris sans une base de sondage sur l'ensemble de la zone d'étude. Les ménages échantillonnés au niveau des producteurs ont été choisis sans aucun critère, mais tout en prenant le soin de distancer ceux-ci pour éviter les effets de répétition. Les questionnaires ont été administrés directement aux producteurs et aux utilisateurs-consommateurs. Cette option a permis de traduire les termes techniques pour amener les enquêtés qui ont le plus souvent un bas niveau de scolarisation à comprendre l'essentiel des questions avant de répondre. Les enseignants, pris sans critère de filière, ni de rang proviennent de l'Université et les chercheurs des centres de recherche du Centre National de la Recherche Scientifique et Technologique (CNRST) situés à Ouagadougou. Avec ceux-ci, les entretiens ont été indirects.

Le choix d'une telle démarche se 
justifie par le fait qu'elle permet d'appréhender concrètement les systèmes en place, les interactions, les rétroactions, les régulations, les organisations, les finalités, la vision globale et les évolutions à l'intérieur de ces systèmes en général. De façon spécifique cette étude s'intéresse aux milieux ruraux et urbains, aux différents domaines d'activité, aux profiles divers et aux intérêts parfois divergents des acteurs ciblés. Ce faisant, la méthodologie sera composée d'étapes successives, les précédentes annonçant les suivantes et permettent à celles-ci de se réaliser convenablement.

\section{Analyse des données}

Les données ont été traités à l'aide de programmes informatiques (SPHYNX, SPSS, STATA et EXCEL). Les résultats sont représentés sous forme de tableaux et de graphiques. Les analyses qualitatives ont été faites à partir des statistiques descriptives.

\section{RESULTATS}

\section{Connaissance des OGM au Burkina}

La connaissance des OGM par les différents acteurs va conditionner leur représentation de ces produits de type nouveau, qui à son tour va influencer leur perception, leur motivation à adopter et leur réaction vis-à-vis de cette technologie. Cette connaissance a été évaluée à travers une série de questions simples qui permet de saisir clairement l'orientation des réponses des personnes enquêtées.

\section{Avez-vous déjà entendu parler des OGM ?}

La plupart des personnes enquêtées a déjà entendu parler des OGM, avec plus de 95\% de oui pour seulement moins de $5 \%$ de non. Les résultats des investigations montrent qu'il n'y a pas une différence signification par rapport au ratio sexe. En effet, 96,98\% des hommes et $91,78 \%$ des femmes ont répondu par oui (Tableau 1).

\section{Quels types d'OGM connaissez-vous?}

Les OGM qui résistent aux attaques des insectes sont évidemment les mieux connus de la population enquêtée. Le Bolgard II est passé par toutes les étapes expérimentales et est depuis la campagne 2008-2009 en production en milieu paysan dans les zones où se sont déroulées les enquêtes. L'introduction de ce coton a fait l'objet d'une vaste campagne de communication. Les autres types d'OGM restent très peu connus, notamment les variétés tolérant les herbicides (Tableau 2).

\section{Canaux d'information et conséquences des OGM}

Il arrive le plus souvent que notre manière de percevoir les choses dépende des informations reçues de la première personne qui a abordé le sujet avec nous. Ceci est d'autant plus vrai, si ce sujet relève d'un domaine complexe pour lequel nous n'avons pas beaucoup de facilités pour obtenir des informations plus détaillées. La biotechnologie et ses applications relèvent de cette catégorie de domaines pour beaucoup d'utilisateurs.

\section{Qui vous a parlés des OGM pour la première fois?}

Selon nos investigations, pour $66 \%$ des répondants, les informateurs ont seulement abordés les aspects positifs des OGM, contre $27 \%$ (Tableau 3) qui ont parlé à la fois des avantages et des inconvénients. Cette manière de passer l'information sur les OGM qui ne comportent pas que des avantages, pourrait avoir influencé le nombre de ceux qui trouvent que les OGM sont une bonne chose.

En poussant les investigations, il ressort que les principaux canaux d'information sont le réseau d'encadrement des sociétés cotonnière $(42 \%$ sur un total de 181 répondants), ce qui reflète l'importance des producteurs de coton dans l'échantillon de l'étude, suivi de l'Internet (34\% sur un total de 181 répondants) qui exprime la participation des enseignants et des chercheurs et des autres acteurs qui ont un niveau d'instruction leur permettant l'utilisation de cet outil moderne. Les radios et les télévisions nationales qui apparaissent comme les meilleurs canaux de diffusion de l'information accessibles pour la majorité des acteurs ne sont pas assez utilisées (Figure 1). 
Quels sont les avantages des OGM?

Les acteurs interrogés perçoivent de nombreux avantages à travers l'utilisation des OGM. En effet, ils sont plus nombreux à considérer par ordre d'importance l'augmentation de la production, la diminution du volume de travail, la diminution des coûts de traitement, l'augmentation de la qualité et la maturité précoce comme principaux avantages des OGM (Figure 2). En prenant ensemble ces avantages, il est possible d'ajouter à ceux-ci des gains financiers. De même, il est possible de déduire à travers la réduction des coûts de traitement, donc des quantités de pesticides, que l'utilisation des OGM comporte des avantages au plan environnement.

\section{Quels sont les inconvénients des OGM?}

Les OGM ne comportent pas seulement des avantages, mais aussi des inconvénients (environnementaux et socio-économiques) spécifiques à chaque milieu et contexte. Selon les producteurs de coton $B t$ rencontrés, il ressort que les principaux inconvénients relèvent des prix élevés et de la qualité des semences, ainsi que de la persistance des attaques parasitaires (Tableau 4).

Tableau 1: Résultats de la question «Avez-vous déjà entendu parler des OGM? ».

\begin{tabular}{lllll}
\hline Question & \multicolumn{2}{l}{ Sexe } & Total \\
\cline { 3 - 4 } & & Masculin & Féminin & \\
\hline Avez-vous déjà entendu parler des OGM? & Oui & 193 & 67 & 260 \\
& Non & 6 & 6 & 12 \\
Total & & $\mathbf{1 9 9}$ & $\mathbf{7 3}$ & $\mathbf{2 7 2}$ \\
\hline
\end{tabular}

Source : Données d'enquête, 2013.

Tableau 12: Types d'OGMs connus par les répondants.

\begin{tabular}{lc}
\hline Types d'OGM & Total \\
\hline OGM qui résistent aux attaques des insectes & 146 \\
OGM qui tolèrent les herbicides & 28 \\
OGM qui ont une teneur améliorée en éléments & 34 \\
OGM qui résistent au stress hydrique & 40 \\
Autres & 8 \\
Total & $\mathbf{2 6 0}$ \\
\hline Source : Données d'enquête, 2013.
\end{tabular}

Source : Données d'enquête, 2013.

Tableau 3: Résultat à la question « Que vous a-t-on parlé des OGM pour la première fois? ».

\begin{tabular}{lc}
\hline Réponse & Total \\
\hline Inconvénients & 17 \\
Avantages & 172 \\
Avantages et inconvénients & 71 \\
Total & $\mathbf{2 6 0}$ \\
\hline Source : Données d'enquête, 2013.
\end{tabular}

Source : Données d'enquête, 2013. 
Tableau 4: Inconvénients des OGM selon les producteurs.

\begin{tabular}{lc}
\hline Selon vous, quels sont les inconvénients des OGM? & Total \\
\hline Prix des semences & 76 \\
Qualité des semences & 15 \\
Attaques parasitaires & 6 \\
Autres & 0 \\
\hline
\end{tabular}

Source : Données d'enquête, 2013

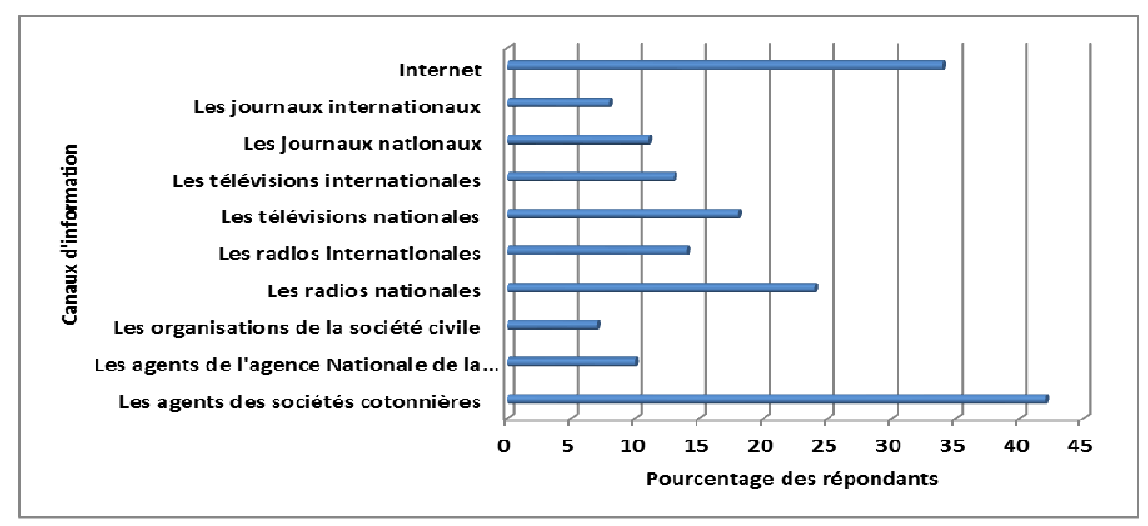

Figure 1: Principaux canaux d'information. Source : Données d'enquête, 2013.

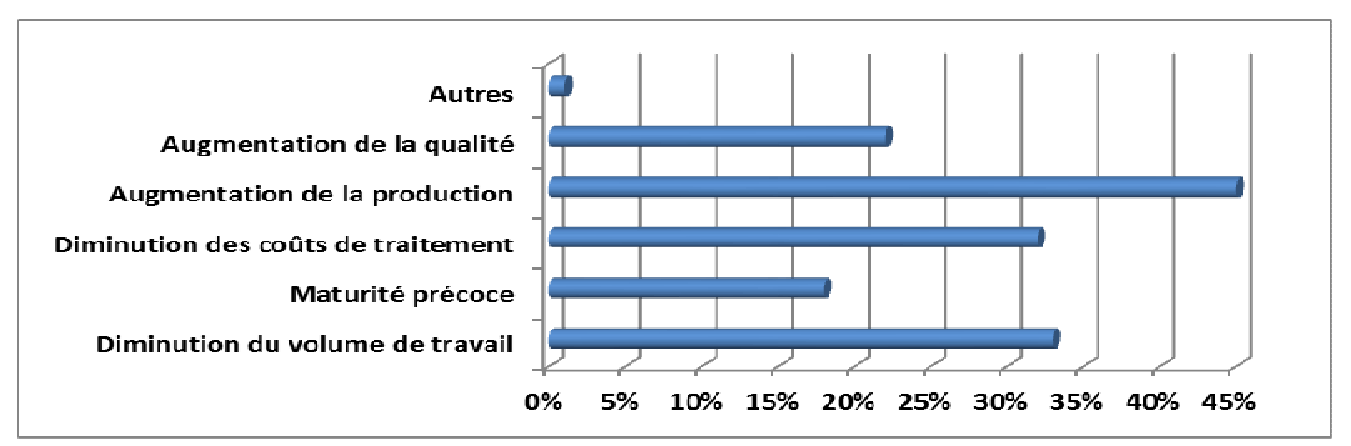

Figure 2: Avantages des OGM perçus par les acteurs interrogés. Source : Données d'enquête, 2013.

\section{DISCUSSION}

Les résultats de l'enquête ont été influencés par le choix de la zone d'étude; d'une part, par le fait qu'elle s'est déroulée dans la ville capitale (Ouagadougou), où le niveau d'éducation est élevé et les moyens de communication développés; d'autre part, elle s'est intéressée au groupe des enseignants et des chercheurs. En plus, dans le milieu rural ciblé, en particulier les provinces du Houet et du Gourma, le coton $B t$ est largement diffusé. $100 \%$ des enseignants et chercheurs et des autres utilisateurs et $67,3 \%$ des producteurs ont déjà entendu parler des OGM. Ces résultats ont été surprenants en tenant compte du niveau d'éducation global du Burkina Faso. En les comparant aux études retrouvées, notamment l'Eurobaromètre 2006 et 
l'Eurobaromètre 73.1 de janvier-février 2010, les pourcentages des Français impliqués dans les investigations qui ont déjà entendu parler des aliments sont respectivement $91 \%$ et $85 \%$ GM (Mary, 2009). Par contre, ils sont comparables à ceux d'une étude similaire menée au Ghana, selon laquelle 100\% des travailleurs relevant des académies et des institutions de recherche, environ $86 \%$ des fonctionnaires et environ $70 \%$ des citoyens ordinaires ont déjà entendu parler des OGM (Buah, 2011). Cependant, l'étude de Oladele et Akinsorotan (2007) a trouvé des résultats beaucoup en deçà au Nigéria avec un groupe d'enseignants et de chercheurs quand ils ont considéré des variétés spécifiques d'OGM tels la patate et le tabac transgéniques qui soignent le choléra et la tuberculose, le riz doré, les variétés de papaye et de maïs tolérant les sols acides, les variétés de céréales et de tubercules qui ont des concentrations ajustées (hausse ou baisse) de nutriments, les variétés qui résistent aux attaques parasitaires, incluant les virus.

En effet, ces résultats peuvent laisser croire que les campagnes anti-OGM menées entre autre par Greenpeace et Biowatch en Afrique et COPAGEN au Burkina Faso n'ont pas eu la même envergure que les actions médiatiques menées par les pro-OGM, et n'ont pas eu un impact considérable à même d'influencer la perception des acteurs (Copagen/Burkina, 2008).

Des études menées en Afrique donnent des résultats comparables. L'étude de Okorie et al. (2014) place l'Internet comme la première source d'information des enseignants et des chercheurs du Nigéria. Quaye et al. (2008) ont trouvé que 78,4\% des enseignants et des chercheurs du Ghana utilisaient l'Internet comme principale source d'information sur la biotechnologie et les OGM. Selon Buah (2011) le bouche-à-oreille est la principale source d'information des Ghanéens ordinaires. Au Kenya, la principale source d'information sur la biotechnologie et les OGM des agriculteurs sont les journaux (43\% de l'échantillon de l'étude), suivis des agents d'encadrement (34\%), puis la radio (12\%) et enfin la télévision (10\%). Pour les consommateurs, il s'agit des journaux (32\%), la radio $(29 \%)$, des agents d'encadrement (27\%) et la télévision (13\%). Pour Parul et Stuti (2011), les médias jouent un rôle capital dans la diffusion de l'information sur les OGM, en particulier dans les milieux où le niveau de connaissance de cette nouvelle technologie est faible et ceci ne manquera d'influencer la perception des populations cibles.

Ces résultats révèlent en partie les nombreux avantages des OGM, qui sont confirmés par d'autres auteurs (Afssa, 2001; Philip et al., 2002; Bennett et al., 2004; Brookes et Barfoot, 2009; Fok et Xu, 2010; Parul et Stuti, 2011; Laibuni et al., 2012). En particulier les études expérimentales menées sur le coton Bt au Burkina (Agbios, 2007; Traore, 2007; Vitale et al., 2007; Traore et al., 2008; Vitale et al., 2008; Ouedraogo et al., 2009; Vitale et al., 2011) ont traité des différents avantages des OGM. D'autres études d'opinion indiquent que la perception des avantages est fonction des intérêts des populations et évolue avec le temps et le niveau d'information (Bonny, 2004). Elle montre l'évolution de la perception des avantages offerts par les OGM aux Etats Unis et en Europe, ainsi que dans d'autres parties du monde.

Gouse (2005) nuance les avantages économiques des Cultures Génétiquement Modifiées (CGM). Cet auteur trouve que les producteurs qui utilisent les semences conventionnelles s'en sortent mieux en année de faible infestation que ceux utilisant les semences transgéniques. Cette étude est en concordance avec les résultats des différentes évaluations économiques réalisées avec le coton Bolgard II. Pour ce qui est du cas particulier du Burkina Faso, les négociations ont permis de terminer des prix relativement intéressants. Néanmoins, les producteurs demandent une baisse des prix des semences. Les principaux argumentaires sont les resemis en cas de mauvaise levée et les incertitudes pouvant apparaître en cours de campagne, ce qui rendrait les charges insupportables. 
Pour Gaskell et al. (2004), 60\% de l'échantillon de l'Eurobaromètre 52.1 croient que les aliments GM n'offrent aucun bénéfice et comportent des risques. Il convient alors de les déceler et de trouver des solutions pour maximiser le bénéfice dans leur utilisation, d'où l'importance de l'évaluation des risques (Gruère et al., 2008).

\section{Conclusion}

Les pro- et les anti-OGM travaillent, chacun selon ses moyens à influencer la perception des OGM. L'Agence nationale de biosécurité contribue à améliorer les connaissances des OGM par les sensibilisations, les émissions radios et télévisées, ainsi que par des articles dans la presse écrite. L'étude monte que plus de $95 \%$ des personnes enquêtées ont déjà entendu parler des OGM. Ce pourcentage élevé vient du fait que l'étude ait été réalisée dans des provinces où le coton $\boldsymbol{B} \boldsymbol{t}$ est en production commerciale depuis 2008. Les variétés génétiquement modifiées les plus connues sont celles résistant aux attaques des insectes, les herbicides tolérantes, celles ayant une teneur améliorée en éléments nutritifs et enfin celles résistant au stress hydrique. Les premiers informateurs ont surtout parlé des avantages des OGM qui sont en autre l'augmentation de la production, la réduction $\mathrm{du}$ volume de travail et des coûts des traitements, ainsi que la qualité nutritive et la maturité précoce. En ce qui concerne les inconvénients, il ressort essentiellement les aspects liés au coût et à la qualité des semences.

\section{CONFLIT D'INTERETS}

Les auteurs déclarent qu'il n'existe pas de conflit d'intérêt sur cet article.

\section{CONTRIBUTIONS DES AUTEURS}

JS est l'instigateur principal de cet article, il a réalisé l'étude, analysé et rédigé le manuscrit (50\%), OMS a participé à l'analyse, à la rédaction et a apporté des amendements au manuscrit (20\%), MS et DECD ont pris part à la conception et à la supervision du travail (15\% chacun).

\section{REFERENCES}

Afssa. 2001. OGM et alimentation : peut-on identifier et évaluer des bénéfices pour la santé ? Étude au travers de 4 exemples : Les plantes résistantes à des insectes, la betterave tolérante au glyphosate, l'enrichissement en vitamine A : cas du riz doré, des microorganismes génétiquement modifiés. France: 67.

Agbios. 2007. Evaluation du risque environnemental associé aux plantes transgéniques avant leur mise en marché : étude cas-témoins basée sur le coton mon 15985.

Bennett RM, Ismael Y, Kambhampati U, Morse S. 2004. Economic Impact of Genetically Modified Cotton in India; University of Reading, Berkshire, UK. AgBioForum, 7(3): 96-100.

Bonny S. 2004. Factors Explaining Opposition to GMOs in France and the rest of Europe. Consumer Acceptance of Genetically Modified Foods. E.R.E. SANTANIELLO. CABI Publishing: Wallingford;169-187.

Bourgou L, Tarpaga WV, Sanfo D, Sawadogo M, Zongo JD. 2014. Préservation de la diversité génétique dans le genre Gossypium au Burkina Faso: collecte et évaluations préliminaires de cotonniers locaux. Int. J. Biol. Chem. Sci., 8(5): 2081-2094.

Brookes G, Barfoot P. 2009. GM Crops: Global Socio-economic and Environmental Impacts 1996-2007. UK.

Buah JN. 2011. Public perception of genetically modified food in Ghana. American Journal of Food Technology, 6(7): 541-554.

Chaklatti-Rousseliere S. 2006. Les biotechnologies agricoles Potentiels et controverses. Grenoble II, Université Pierre Mendes France. Thèse de 
Doctorat en Sciences Economiques, $278 \mathrm{p}$.

Copagen/Burkina. 2008. Déclaration de la COPAGEN - BURKINA sur l'introduction et la généralisation de la culture du coton transgénique au Burkina, Semences de la biodiversité.

Degla KP. 2012. Rentabilité économique et financière des exploitations cotonnières basées sur la Gestion Intégrée de la Fertilité des Sols et des Ravageurs au Nord-Bénin. Bulletin de la Recherche Agronomique du Bénin, Numéro spécial Coton: 1840-7099.

Fok M, Xu N. 2010. L'intégration technologique et le développement du secteur semencier Deux facteurs de la diffusion du coton-Bt dans la vallée du fleuve Yangtsé. Économie rurale, 317: 40-56.

Gaskell G, Allum N, Wagner W, Kronberger $\mathrm{N}$, Torgersen H, Hampel J, Bardes J. 2004. GM foods and the misperception of risk perception Risk Analysis, 24: 185-194.

Gnankiné O, Traoré D, Sanon A, Traoré NS, Ouedraogo PA. 2007. Traitements insecticides et dynamique des populations de Bemisia tabaci Gennadius en culture cotonnière au Burkina Faso. Cahiers Agricultures, 16(2): 101-109.

Gouse M. 2005. Aspects of biotechnology and genetically modified crops in South Africa Department of Agricultural Economics, Extension and Rural Development. Bellagio, 28.

Griffon M. 2010. Comment nourrir neuf milliards de personnes sans détruire l'environnement? Annales des Mines Responsabilité et Environnement, 2(58): 58-61.

Gruère GP, Mehta-Bhatt P, Sengupta D. 2008. Bt Cotton and Farmer Suicides in India Reviewing the Evidence. IFPRI Discussion Paper, 64.

Houndete TA, Hougni A, Aladji S, Dagoudo A, Zoumarou-Wallis N, Thomas-Odjo AA. 2015. Comportement de principaux bioagresseurs et maladies du cotonnier sur les variétés éprouvées de cotonnier (Gossypium hirsutum) sous différentes doses d'engrais à Angaradébou au Bénin. Int. J. Biol. Chem. Sci., 9(1): 217- 224.

Kahn A. 2005. Biotechnologies, Progrès, Les défis de l'agriculture au XXIe siècle. Leçons inaugurales du Groupe ESA: 38.

Koulibaly B, Dakuo D, Traoré M, Traoré O, Nacro HB, Lompo F, Sedogo M. 2016. Effets de la fertilisation potassique des sols ferrugineux tropicaux sur la nutrition minérale et la productivité du cotonnier (Gossypium hirsutum L.) au Burkina Faso. Int. J. Biol. Chem. Sci., 10(2): 722-736.

Koulibaly B, Traore O, Dakuo D, Lalsaga R, Lompo F, Zombre NP. 2013. Acidification des sols ferrugineux et ferrallitiques dans les systèmes de production cotonnière au Burkina Faso. Int. J. Biol. Chem. Sci., 8(6): 28792890.

Laibuni NM, Miriti LC, Waturu CN, Wessels W, Njinju SM. 2012. Cost benefit analysis of transgenic cotton containing Cry1 AC and cry2AB2 genes and hart 89M: evidence from confined field trials in Kenya. African Journal of Horticultural Science, 6: 27- 34.

Mary S. 2009. La perception des OGM par les consommateurs: une étude comparative exploratoire entre la France, le Québec et le Canada anglophone, Université du Québec à Montréal, 299.

Okorie NP, Marshall MJ, Akpa MO, Ademowo GO. 2014. Perceptions and recommendations by scientists for a potential release of genetically modified mosquitoes. Nigeria Malaria Journal, 8.

Oladele OI, Akinsorotan OA. 2007. The attitude towards genetically modified organisms (GMO's) and their effect on health and environment in southwestern Nigeria: scientists' perception. Journal 
of Central European Agriculture, 8(1): 91-98.

Ouattara FS. 2011. Quel bilan après trois années d'expérimentation ? Les paysans du Burkina veulent une véritable politique agricole, défis sud.

Ouedraogo SL, Sanfo D, Somda I, Tiemtore BC. 2009. Analyse de l'influence du fonds génétique, des conditions climatiques et du mode de protection phytosanitaire sur l'expression de la bactériose chez différentes variétés de cotonnier au Burkina Faso. Tropicultura, 27(1): 31-34.

Parul G, Stuti G. 2011. Factors Influencing Public Perception: Genetically Modified Organisms. GMO Biosafety Research, 2(1): 1-11.

Philip JD, Belinda C, Eliana MGF. 2002. Potential for environmental impacts of transgenic crops. Nature Biotechnology, 20: 567-574.

Quaye W, Yawson I, Yawson RM, Entsi Williams I. 2008. A Stakeholder Approach to Investigating Public Perception and Attitudes towards Agricultural Biotechnology in Ghana. Tailoring Biotechnologies, 4: 55-70.

Rule S, Langa Z. 2005. HSRC client survey. Public Understanding of Biotechnology, 32.

Traore O. 2007. Recherche mise en œuvre avant et pendant la culture du coton $\mathrm{Bt}$, évaluation et gestion de l'impact : Cas du Burkina Faso. Consultation d'experts sur la biotechnologie appliquée au coton, Ouagadougou, 45p.
Traore O, Denys S, Vitale J, Traore K, Bazoumana K. 2008. Testing the Efficacy and Economic Potential of Bollgard II under Burkina Faso Cropping Conditions. The Journal of Cotton Science, 12: 87-98.

Umezawa T, Fujita M, Fujita Y, YamaguchiShinozaki K, Shinozaki K. 2006. Engineering drought tolerance in plants: discovering and tailoring genes to unlock the future. Plant Biotechnology/Food Biotechnology, 17(2): 113-122.

Vitale J, Boyer T, Uaiene R, Sanders JH. 2007. The Economic Impacts of Introducing $\mathrm{Bt}$ Technology in Smallholder Cotton Production Systems of West Africa: A Case Study from Mali. Ag.Bio. Forum, 10(2): 7184.

Vitale J, Glick H, Greenplate J, Abdennadher M, Traore O. 2008. Second-Generation Bt Cotton Field Trials in Burkina Faso: Analyzing the Potential Benefits to West African Farmers. Crop Science, 48: 9.

Vitale J, Ouattara M, Vognan G. 2011. Enhancing Sustainability of Cotton Production Systems in West Africa: A Summary of Empirical Evidence from Burkina Faso. Sustainability, 3: 11361169. 